\title{
Giittoriider lleberblit.
}

Sa bie Erofkntud aus einer Summe von Erfenntnilīen weiteht, ju benen alle europäifden sölfer einen Theil beige: tragen baben, io gewährt igre Beichichle Belegenheit zu ipannen= ben $\mathfrak{B}$ ergleifjen, benn in ber $\mathfrak{A}$ trt ihrer Reijtungen ipiegeln iid) fomobl ber Benius als aud bie politiichen Echiffiale ber ein= jelnen \$ölfer mieder. So gehört bas fabolaftiid)e Mittelalter, obgleich ieine brei gröpten \$hyjifter, Ullbert v. Bolitäot ein Deutider, Bincentins $\mathfrak{n}$. Weaupais ein Franzofe, Moger Baco ein Brite maren, ganz intidieoen bell Stalienern an, welde feitbem mehr uno mehr veridgminden. In ber Beit von Mlegiomontan bis au Repler find bie Deutiden weit allen anbern Rationen üherlegen; bod cntwidelt jid bie holländoilde Sdaule unter 21nregunty von fremer (Mercator) unto Dertel (Drteliuas) idon um bie Mitte beș 16. Jahbrhunderts. Ceit bem Jahbre 1669 vereinigt ïh) aller (Slanis aui ßaris und verneilt bort

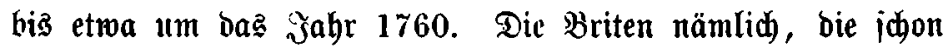

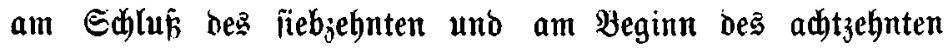

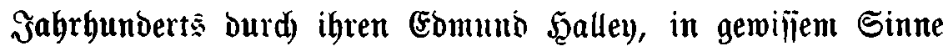
aud burch Hewton io glüctlid vertieten wurben, errüllen in der zameiten Şälfte meb̧r uno mehr ber Borbergrund bes acht=

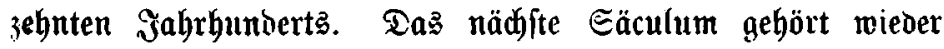

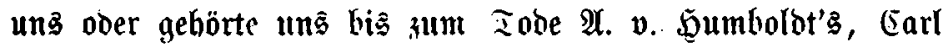




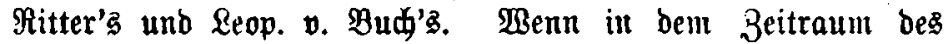
fiebzehnten Jaabrhundertă, ber zwiłden Siepler uno Seibniz liegt, beutiche Ramen biz auj einen einzigen in unjerer Beidjidyte

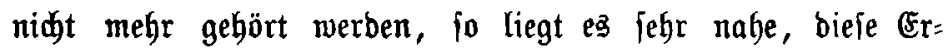

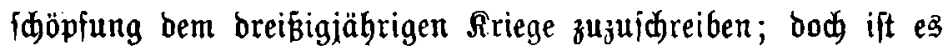
hödit bebeut fam, baß gerabe jene ßeit aud für bie Sdameiz ein tobter Maum gewejen ift, benn wie Stuber beobadfet hat, herridte bort feit Beßnner's Tode eine geiłtige Errftarrung uno

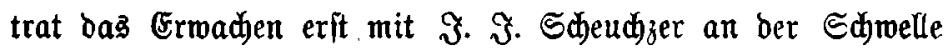

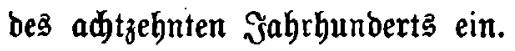

Beginut bie Erofunde mit ber $\mathfrak{A}$ bideibung bes Trodenelr und Flüffigen, burd bie Entbectungen ber Seefahrer, wo bie

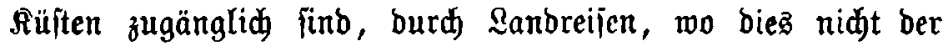

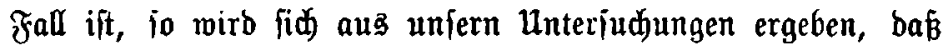
bie räumlidfe Ermeiterung bes $\mathfrak{B}$ Biffens in ber älteren Beit bis 1650 gewiffen Befeben gefordste. So war baz felb ber \{panifiben Entbeçungen burdh bas SBorfommen ber eblen Metalle

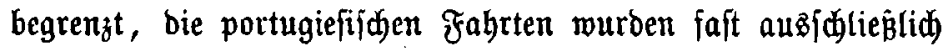
nad) ben Bemürzläubern geriftet, bas Borbringen ber $\mathfrak{R u f f e r ~}$ eriđjien abyängig von ber Berbreitung ber ßelzthiere, unto nur non ben Briten barf man fagen, baß́ fie bei igren Entbefungen ein höheres 3iel, bie Bertürzung ber Seeroege, im 2 uge be= hielten. Die Deutiden, melde früber zu allen Zeiten Sdjiffe, $\mathfrak{z} \mathfrak{u}$ feiner eine Flotte, bejaß̧en, fonnten fonft hödfftens ala $\mathfrak{3}^{\mathfrak{u}}=$ fdauer an fremben Thaten Theil nebmen, wie Tyrfer, welder bie Morntannen nad Birginien; Martin Behaim, ber Diogo (Eam nady 2 Angola; Steller, ber Bering auf ber Fahrt zur Entbecưng Âmerifas; bie beiben Foriter, bie Coof nach bem Süb:

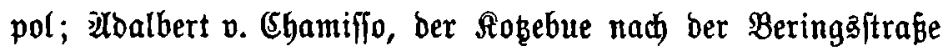
begleitete. Mir müffen unz tröften mit ben franzojen, bie zmar eine Seemadt, aher feine Entbecfer erften Manges bejaken,

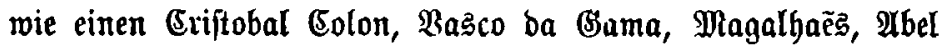
Taşman unb รamesె Coof.

Die beneibenazmerthen Berbienfte um unjere Biffenidaft, 
welde bie granioien feit 1669 jich gefichert haben, gründen

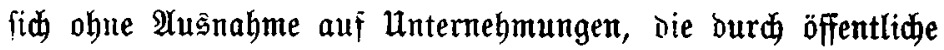

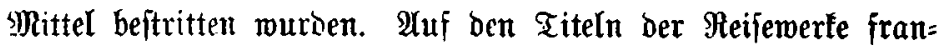
zöfificher Belehrter lehren jtets bie $\mathfrak{B o r t e}$ wieber: Voyage fait

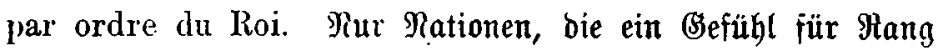
und (S)rößze befiķen, werben ben nötbigen 2ufmand bemilligen,

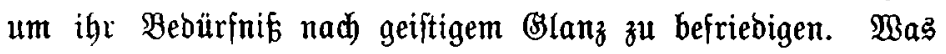
beutiche Staaten geteiftet haben, läßst fich mit beidäämender

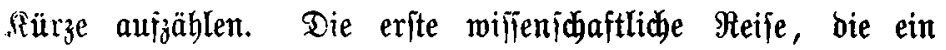
beutfdyer Monard auşühtren lies, war bie Cendung von Spix uno Martius nady Brafilien. Breunen beftritt einen Theil ber Meijefoftcn für Şemprid) und Ehrenberg, e己 bemilligte bent

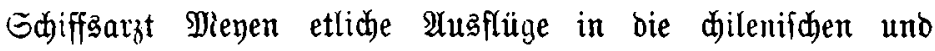
bolivianifđen $\mathfrak{A}$ nowen unb veriah ben jüngern Sdjomburgl mit

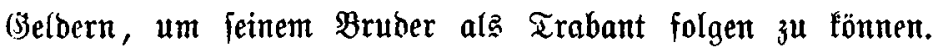
2huberbem bleibt nur nod) bie Eridaffing eineả \&ebrftuhles für Carl $\Re$ itter in Berlin übrig, benn nicht einmal fo viel

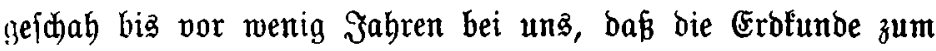
Rehrgegenftand an unfern Şochidulen erboben worben wäre, weaghalb auț biz auf ben heutigen Tag nod ber geographifde

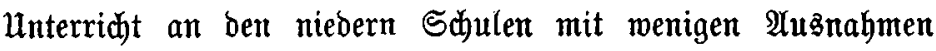
uno trok ber portrefflicken ḩandbücher auf berjelben traurigen Etuje fteht wie im Эabre 1723, ałą Şühner burd jeine "Beographifden Fragen" bie fujon non \$liniuz verabftheuten locorum nuda nomina ala freubelofe (sedädbtnißß̧belaftung ber Gugend ben Rehrern überlieferte.

Fehlt es una, moran Franzofen und Briten fo reidj findo, an nationalen Thaten zur Beförberung bea Bifjena, an foldyen beneibenswerthen Unternebmungen, wie bie Cenbung Şallen's in baz atlantif(d)e Meer, Bouguer's und Raconbamine's nach) ßeru gewejen find, fo war bafür bei uns bie Opferluft ber (Einzelnen um fo regfamer, einea Âtex. v. Şumboldt, Leop.

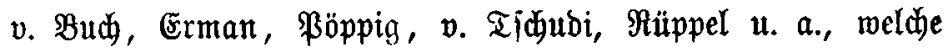
im Dienjt ber Bifferridaft theils entbehrten, theila heträdtlide 


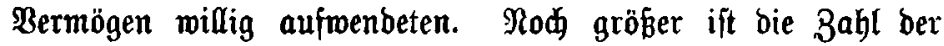
Deutiden im Solbe frember Megierungen. ${ }^{1}$

Das Bebiet ber mathematifden Beographie murbe von beuticher Beiftealtraft beherridgt, in ber Beit, wo Namen Elangen wie Regiomontan, Berner, bie beiben Bienemib̨, Copernicus̄

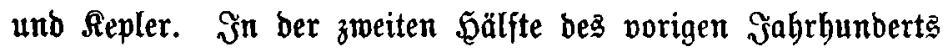
zeidneten fid) nur unjer Lambert und Tobias Mianer, ber Re= formator ber Monotafelı, aus, welder lebetere jelbft flagt, baß zu feiner 3eit in fremben $\mathfrak{B e l t t b e i l e n ~ m e b r ~ g e f i c h e r t e ~ D r t a : ~}$ beftimmungen vorbanben maren als in Deutidilanb! Seit Repler baben fich um bie $\mathfrak{A}$ ußbiloung ber mathematif(t)en Beo= graphie faft auşdjięlid mur Franzofen Berbienite erworben. Sidjt nur verbanten wir ignen bie Einfübrung und erfte $\mathfrak{A} n=$

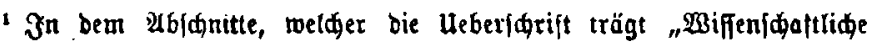
Reifen und wiffenidaftlide Entbeder" baben wir nid)t eine firenge $\mathfrak{U b}=$ fonberung ber Stoffe, welde ber (Beidjidte ber (Erblumbe, von benen, welde ber Beididte ber \&änberbejфreibung angebören, beobaфtet. Mls ber plan zur "Befdidte der Wiffenjdaften" entrorfen rutbe, hatte ibr erhabener

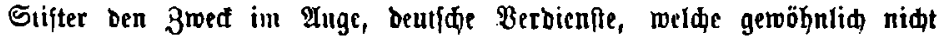
fonosl aus Meib ober Uebelwollen, fonbern aus unbetanntihaft mit unjerer fdrwierigen Sprade bon ben fremben miß̧tannt merben, ber Bergeffengeit zu entreízen. So gei由ah es benn, baj in jenem Ib/dritt aud folde Irbeiten bon Deutfden unb Deutfdruffen beridfidtigt wurben, bie nur ber Bejdidite ber Eborographie angebören. Fer ungetwarnte Refer tönnte

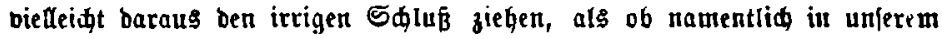

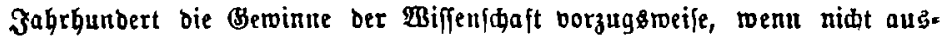

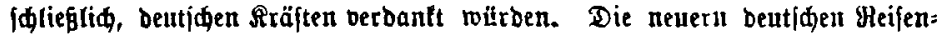
ben füllen allerbings butd ibre bielfeitige Bildung einen febr bebeutenten Maum in ber Befdidte bes neunzebnten Jahrhunderts aus, einen Ber gleid ihrer Reifing

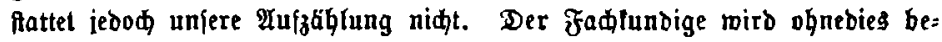
merten, ¿aß in unferem Berzeidniffe die grojartigen Urbeiten der fatho=

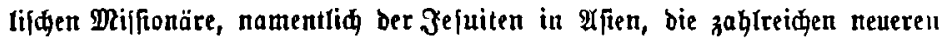
franzörfiden unb ruffifden croumiegelungen fajt gänżlid fehlen, ocr ipanifden Unternehunungen nut fü̈tig gebadt, bie Berbienfte foldcr Reifenden, wie Eaillié und (Eailliaud, Ealt, Bruce, Burdharbt, Sadtier,

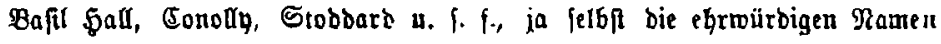
eines Mungo Bart unt arleranber Burnes gar nidte soer nur porïber= gebend ecwäh̆nt rocrben. 
wenbung ber \&ängenbeftimmungen nađ̆ ben Berfinfterungen ober Beleuchtungen ber Supiterämonbe, fonbern fie hatten

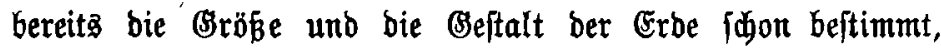

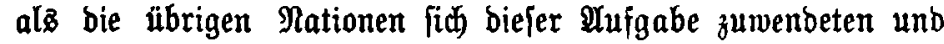
nidłts anderez zu leiften übrig fanden als bie Berfdärrfuna

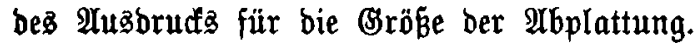

Abfolute $\mathfrak{t o ̈ b e n ~ v o n ~} \mathfrak{D e r g g i p f e l n}$ Eonnten burd Dreiecte zuerit nur in Frantreid gemeffen merben, aber aud bie baro=

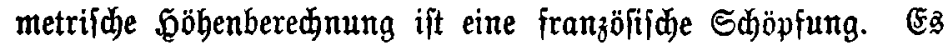

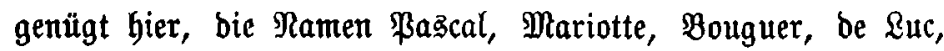
Ramond und Laplace zu nennen, benn ebenjo wie wir bie beutjden Sdjweizer zu ben Ulnirigen rednen, müffen wir aud

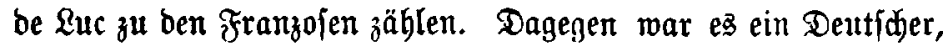
nämlidy Illeranber $\mathfrak{v}$. Şumboldt, weldyer zuerfit aus ben all= mählid fich bäufenden Šöhenbejtimmungen bie vergleidjende

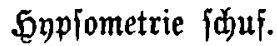

Beit gröber find bie Berbienfte ber Deutfden um bie $\mathfrak{F r}=$ fenntniß̧ vom innern Bau ber Erbrinbe. Wenn man bez̧ Dänen Steno uno Seibutizend frühreife 2 nfidten abrednet, fo verbanten wir Werner allein burd) SUfftellung bea frormations: begriffes bie Brunolage und alle Fortidritte ber Beologie bis zu ber 3eit, wo nad ben \&agerungäberhältniffen aud bie ein= gefdloffenen Beriteinerungen gleidzzeitig in England und in Frantreich zur dronometrifđ)ent Beftimmung ber Felzarten her= beigezogen wurben. Das Bidhtigite, was man bis jebt über ben Bau und bie georbnete Lage ber Bultane weis, verbanlt

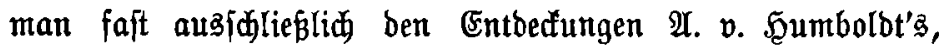
\&. v. Buct's und Franz 3 unghubn's.

Es verftand fid von felbft, bas eine jeefahrende Sation wie bie Briten am frübeften ben $\mathfrak{A} n t r i e b$ füblen muß̣te, bie Nätbjelipradje ber Magnetnabeln zu entziffern. WBenn man

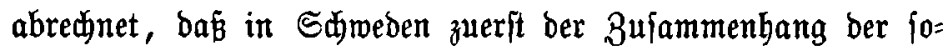
genannten magnetifdjen Bewitter mit bem Renthten ber man-

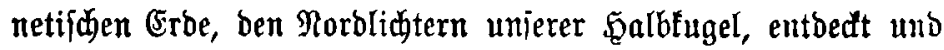


bort bie erften gleidzeitigen Beobadtungen verabrebet wurben, fo find faft alle midjtigeren Bejeke ber magnetijhen Erofräfte, bie Senfungäerideinungen ber Miagnetnabel, bie jeculären Ber= änberungen uno bie tägliđhen Sdbanfungen ber Mißßpmeifung in Englano gefunben und ebenjalls bort bie eriten magnetifajen Rarten entmorien worben. Dod hat fich Scumbolot unver=

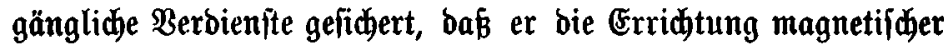
Şütten bis nad) Befing veraullaßste uno baß er am frübeften bie ungleidje Bertbeilung ber Intenfität befannt madjte, für weldhe erft Gauk $\delta a \mathfrak{s}$ abjolute Maß finden lehrte.

Die älteften Seetiefenmeffungen und bic älteften Seetiefen=

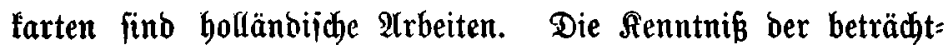
lidjiten Meereşitrömungen verbanft man bagegen fpanijden unb portugiefiichen, einige aud englifhen \&ootien, bod wurbe bas erfte plyyiffaliiłde Bemälbe biefer Eridgeinungen lange por Sralley's Binbtarte in Deutiblano entworfen. Die Albhängig= feit ber rhythmiichen Shtwanfungen bez Eeefpiegela von ber 3ugfraft bes Monbes hat Repler vor Remton außgeiproden, aber bie tiefere Begrünoung ber Rehre und bie Darftellung von Fluterfdeinungen auf Beltfarten find britifde Berbienfte, ‘benio wie bie Erfenntnis ber oceanifhen Tiefentemperaturen.

Bie bie franzofen und bie franzöjijichen Edameizer zuerit ben Drud bes sufttreifes beftimmten, fo haben fit aud bas Befte gefunden, was mir von ben barin fdyebenden $\mathfrak{B a f f e r}=$ dämpfen wifīen. $3 \mathfrak{u}$ bem, was Leroy lehrte, was Saufinure zurrft gentefien und \$ictet beobadjtet bat, ift jebr wenig binzugefügt morben; in Dentidfand murbe nur bie befte pindaro= metrifbe Formel gefunden. Erift Şalley vermodte bie Erfdei= unug ber Monfune zu erflären, wie er auch zuerít theoretifích das̄ Dajein eines rüfllaufenton ßaîatez geforbert hat, bèr aber unfichtbar Glieb, bis ihn Seop. vo. Buch's jđarfes 2luge in ben $\mathfrak{B}$ itterungảerideinungen ain $\Re_{i c}$ von Teybe erfannte.

Ueberall, wo es etmas zu meffen gab, Gaben wir bie

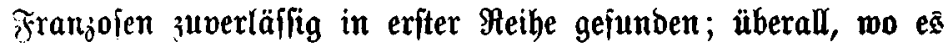


galt, burd Bergleidje ber angebäuften Meffungen zu höberen Wabrbeiten unb Beferen fid zu erheben, hegegnen wir meiftens ben Deutiden. Das Drebbel'\{dje \&uftthermometer, von ber Academia del Cimento in ein Beingeiftthermometer vermanbelt, er= bielt eine Scala, beren $\mathfrak{B e r t h e}$ fid vergleidgen ließen, erft burd Reaumur. Benn man auch bie veritänbige Benußung biejez Sulftruntente $u$ um bie Mitte bes vorigen Jahrhunberts in Upiala iđon eingejehen hatte, jo barf man bod) bas (Beburts jahr ber Meteorologie nidjt vor 1780 fezen, wo ein bayerifder Fürft bie berühmte mannheimer Befellfdaft ftiftete. Selbft bann nod blieben bie thermometrifden Beobadtungen, bie feitbem fich anbäuften, todte Werthe, biz fie $\mathfrak{A}$. v. Sumboldt 1817 plöglich burd) Begrünbung ber mathematifden $\mathfrak{A l i m a t o =}$ Iogie belebte unb biefe zu einem ber widtigiten fä̈her ber phyfifalifden Erofunbe erhob, denn gewiés ift nad ber mathe= matijaen uno hypjometrifden Lage eines Drtes feine ifotherme Beftimmung baz Ent\}丨eibungåvollfte.

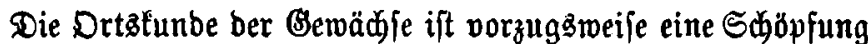

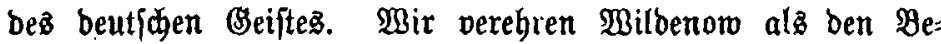
grünber ber $\mathfrak{A} r t e n f t a t i f t i f$, sumbolbt, Reopolb $\mathfrak{v}$. Bud und ben Sdyeden $\mathfrak{B a b l e n b e r g}$ ala die Sdjopfer ber Pflanzenflimatologie, Earl Mitter als ben Berfaffer ber erften botanifden אarte. Rad)bem Treviranuz unb Mobert Brown bas Bejek für bie veridiebenartige Berbreitung ber niebern, böhern und voll= fommenften Bemädfe feftgeftellt und be canbolle bas phyfio=

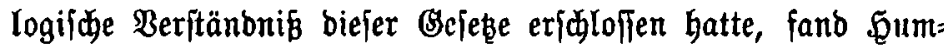
boldt bie erften Thatfachen über bie Berbreitung ber Framilien unb bie Banberungen ber Bewädje, fo baß ber Däne Shoum über hinreidjenbe Borarbeiten verfügte, alz er bie Brunbzüge biejer jungen $\mathfrak{B i f f e n}$ idjaft entwarf.

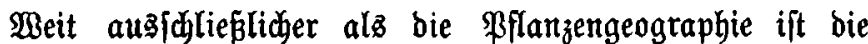
Ortäfunde ber Thiere ein beutiches frad) gemejen. Rur burd feinen groben Borgänger Buffon unterftübt entwarf Bimmer= mann bie erfte Meltfarte für bie Säugethiere. Treviranus 
erweiterte die von ihnt betretene $\mathfrak{B a b n}$, Jlliger begründete bie

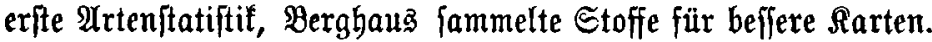

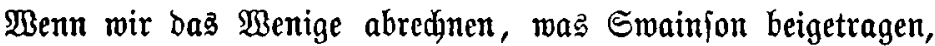

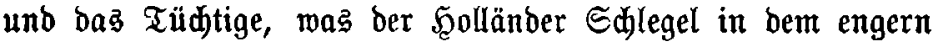
Bebiete ber Seerpetologie burdjgejübrt hat, fo finden wir nur

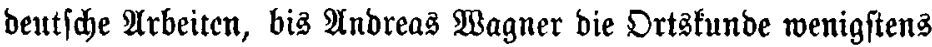
Der Eäugetbiere auf bicjenige Stufe erhob, weldje bie Pflanzen= geographie zu Sd)ouw's Beit bereits erreidyt hatte.

Ein niederlänbifdyer $\mathfrak{A}$ natom erfand baß erite Berfahren, unteriđjiebe im $\mathfrak{B a u}$ ber Menjdenfdäbel zu mefien, aber ben

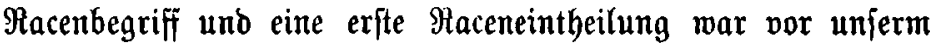
groben Blumenbad) nimt vorbanden. Wit ibm beginnt bie Anthropologie als Biffenfdjaft, bie aud jeitbem, wenn aud

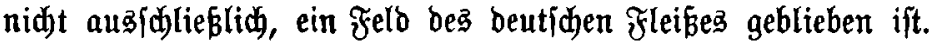
Die frühefte Elaffificirung ber ihrer Spradie verbantt man ben ânregungen Reibnizenz und bem Sammlerfleiße Satharineñ ber Broken; aber baß ridtige Berfahren beim Bergleid führte ber älteitte Meifter polyglotter Singuiftif, Don Lorenzo Servís ein. Die Spradjenvermandt= \{daft ber Briechen uno Römer mit ber Sanffrit rebenden Bevölferung bea alten Snbiena war fidon gegen cnde bea vorigen Jahrhunbertz ben Mitgliebern ber lonboner afiatijajen Bejelffdaft fein Beheimnißs mehr, allein die Senntnis einer indogermanifden Spradjenfamilie perbanten wir erft Friebrid) Sablegel und inge ftrenge grammatifde Begrünoung unjerem Franz Bopp.

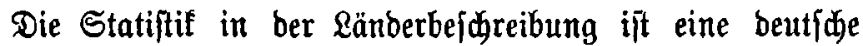
Sd)öpfung Mdenmall's; niemanb vor Siísmild batte ein Mittel gefunden, Bolfşa bienft ift eas, zuerift bie Beftinumung ber Bevölferungabichtigleit als eine geographifdje Ilufgabe erfaß̧t und gelöst z̆u baben.

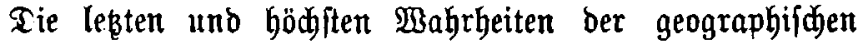

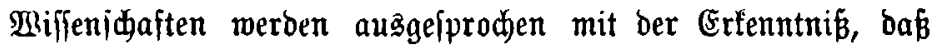
ber Bau-ber Ėrobberfläde und bie von ibm abjängigen Ber: 


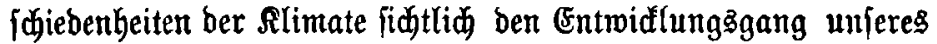

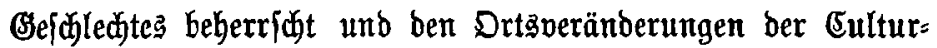
fike ihren ßfab abgeftedft haben, fo bas ber Inblid ber Ero= gemälbe unz bahin füfrt, in ber Bertheilung von Ranb und Baffer, von Ebbenen unb b̧öhen eine von IInjang gegebene ober wenn man will beabjidjtigte Wenbung menjwlider Be= ¡dide zu burdjidauen. Seit Strabo biz auf unjer Sabrbun= bert war niemand biefen tiefen Beheimniffen näber getreten. Auber biefen vielen finnigen Bsebanfen, bie $\mathfrak{A}$. v. hrumbolot aużgefprođen ober mittelbar angeregt hat, lamen bie grö̈ten Offenbarungen aus bem Munde Earl Aitter's, von bem man wohl fagen tann, er habe die naturmiffenfidaftlide Erofunbe bejeelt, er habe zuerit in bem atntlite ber einzelnen welt= theile, welde er bie groben 3 ndivibuen ber Erbe ge=

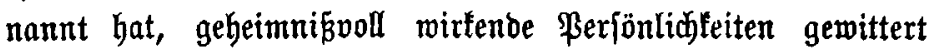
ober wenigitens bod ifre Berridutungen in ber Beididfte

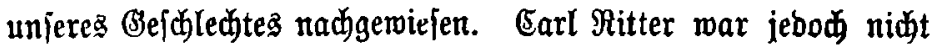
ohne Borgänger, fondern wir merben vielmehr zeigen, baß fäon in ber Edjule, meldje (Satterer begründete uno zu ber

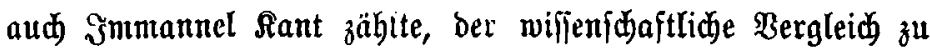
ben Rieblingä̈̈bungen beutjder Beographen gebörte.

Ber bie Befojidgte der Erbfunde zur Sand nimmt, um

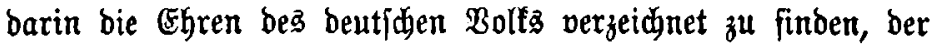
wirb gemifotten Eindrüđen entgegengehen. Er witb gewahren, baß er einer Nation angeböre, bie überreid an 3ierben unb arm an Thaten ift. Wo hobe aufgaben nur burw bie Rräfte eines Staates gelöst werben fönnen, zeigt unjere Be=

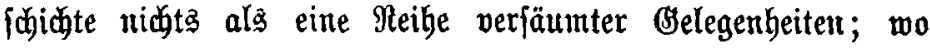
e马s aber bem Einzelnell möglid war, ohne öffentlichen Beiftand ber Biffenfdjaft grofe Diemfte zu leiften ober no frembe Mationen thatenluftig nach Werfzeugeu judten, ba baben fid

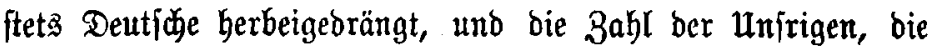
in bie Befahr gingell und in ifgr unterlagen, ift bis auf bie Begenwart ruhmmüroig groß gewefen. Was hätten anbere 
Rationen geleiftet, wenn fie über eine ähnlide $\mathfrak{F}$ illle geiftiger Sräfte zu verfügen gehabt hätten! Menn mir oennod bei ber Bertbeilung ber miffenidaftlidjen Berbienfte nidjt hinter anbern Böltern zurüđftehen, fo müffen wir unfere Bertreter um fo böher feiern, weil fie fo viel erringen tonnten, obgleid) fie Deutjđe waren! 